\title{
Análisis de la vulnerabilidad social en estudiantes del Nivel Medio Superior en el estado de Hidalgo (México): desde la pandemia de COVID-19 a partir de las políticas públicas
}

\section{Analysis of social vulnerability in upper secondary level students in the State of Hidalgo from the COVID-19 pandemic a look at public policies}

IBARRA RAMÍREZ, Adriana A. ${ }^{1}$

CÁCERES MESA, Maritza L. ${ }^{2}$

DOMÍNGUEZ JIMÉNEZ, Ismael ${ }^{3}$

\begin{abstract}
Resumen
La pandemia COVID- 19 ha generado cambios en lo social, laboral y educación. Esto pone en desventaja social a la población más pobre. En esta investigación de corte cuantitativo y exploratorio se analiza la situación de vulnerabilidad social de estudiantes del nivel medio superior en dos bachilleratos en Hidalgo, a partir de políticas públicas implementadas por el gobierno federal y estatal. Los resultados descriptivos muestran el $34 \%$ de las familias de los estudiantes tienen carencias económicas y el $20 \%$ desempleo.
\end{abstract}

Palabras clave: vulnerabilidad social, políticas públicas, COVID -19.

\begin{abstract}
The COVID-19 pandemic has generated changes in social, labor and education. This puts the poorest population at a social disadvantage. In this quantitative and exploratory research, the situation of social vulnerability of school students in two high schools in Hidalgo is analyzed based on public policies implemented by the federal and state governments. Descriptive results show $34 \%$ of the students' families have economic deprivation and $20 \%$ unemployment

Keywords: social vulnerability, public policies, COVID-19.
\end{abstract}

\section{Introducción}

La pandemia derivada por el COVID-19 está afectando a la comunidad global, ha colapsado los sistemas de salud, ha desmantelado las economías, las escuelas han cerrado en su totalidad y los gobiernos de todo el mundo se han paralizado, pues todas las decisiones están condicionadas en mitigar la propagación del coronavirus por su efecto invasivo en la salud humana, lo que ha condicionado como medida extraordinaria, que la mayoría de los estudiantes confinados están aprendiendo desde casa (Sanz, Sáinz, \& Capilla, 2020). En particular las aulas en todos los sistemas educativos transitaron súbitamente a los hogares, adoptando la modalidad de educación a distancia, mediadas por uso de las Tecnologías de la Información y la comunicación (TIC), proceso que, por su

\footnotetext{
${ }^{1}$ Estudiante del Doctorado en Políticas Públicas. Colegio de posgrado. Universidad Autónoma del Estado de Hidalgo.ib122374@uaeh.edu.mx 2 Jefe del Área Académica de Ciencias de Educación y Profesora Investigadora. Universidad Autónoma del Estado de Hidalgo.

maritza_caceres3337@uaeh.edu.mx

${ }^{3}$ Investigador. Maestría en Tecnologías para la Información en la Educación. Universidad Autónoma del Estado de Hidalgo. ismael@uaeh.edu.mx
} 
emergencia, no permitió prever cómo podían influir los factores socioeducativos en el aprendizaje de los estudiantes.

A partir de la situación epidemiológica, que provocó la pandemia global por el coronavirus SARS Cov-2, los estudiantes y docentes han ido adaptando sus metodologías a esta modalidad, haciendo uso de los espacios que disponen en sus hogares y a utilizar los dispositivos electrónicos y la red de internet, si es que existe, para desarrollar sus actividades académicas; situación que según la Organización para la Cooperación y el Desarrollo Económico (OCDE, 2020), requieren de un análisis contextualizado en función de las necesidades, problemáticas y oportunidades, para atender en la medida de lo posible las demandas de la comunidad educativa y generar estrategias para la atención especial a estudiantes más vulnerables, en función de evitar el aumento de las desigualdades educativas y sociales.

En este estudio se aborda, el estado que guarda la vulnerabilidad social con respecto a las políticas públicas implementadas por el gobierno federal y estatal a través de las Becas para el Bienestar Benito Juárez y con la aplicación móvil (App) SIDEMS respectivamente. El análisis se enfoca en alumnos del Colegio de Bachilleres del Estado de Hidalgo (COBAEH) de Tepehuacán de Guerrero, ubicado en la Sierra Alta entre los límites de Hidalgo y San Luis Potosí, con difícil acceso debido a sus características geográficas; y el plantel COBAEH San Agustín Tlaxiaca que se encuentre ubicado en la zona metropolitana de la capital hidalguense y su acceso es más viable por su ubicación en el contexto urbano. En este contexto es importante precisar que la beca Benito Juárez, es la que actualmente se proporciona a estudiantes que viven en condiciones vulnerables y aplica desde la educación básica hasta el nivel superior, en el caso del nivel medio superior está dirigida para "asegurar la permanencia" de los estudiantes y por el gobierno federal, con un apoyo de 800 pesos mexicanos mensuales, durante 10 meses que corresponde a la duración del ciclo escolar y precisamente atiende a grupos vulnerables que se encuentran en pobreza (Coordinación Nacional de Becas para el Bienestar, 2020). Por otra parte, se ha fomentado el uso de la App SIDEMS por el gobierno estatal, a partir de la pandemia COVID-19, desde agosto del 2020 para atender la economía de las familias hidalguenses y elevar los indicadores de calidad y permanencia educativa. Los estudiantes descargan la aplicación en su celular y pueden acceder a los contenidos de aprendizajes, sin conexión continua a internet (SIDEMS, 2020).

En este ámbito se analiza la situación considerando la Reforma Integral de la Educación Media Superior (RIEMS), se describen los retos de la situación que prevalecen y sus actores, posteriormente se estudia el término de vulnerabilidad social y políticas públicas, para finalmente dar a conocer la metodología utilizada, los resultados y las conclusiones. En una segunda etapa se aplica un modelo estadístico en función de poder comprobar o rechazar la teoría provisional de esta investigación, la cual se sustenta en un estudio de corte descriptivo e inferencial, basado en una prueba de hipótesis con la curva normal debido al gran tamaño de la muestra. Se considera como $\mathrm{H}_{0}$ : A partir del apoyo de becas y la utilización de SIDEMS durante la pandemia COVID19 se ha reducido la vulnerabilidad social en estudiantes de la educación media superior en Hidalgo. Se presentan resultados descriptivos, como referentes que permiten la toma de decisiones académicas en el contexto de estudio.

Se ha considerado el término vulnerabilidad, de acuerdo con Busso (2001) citado por Rosas Arellano y Bernal (2019), es definida como el "riesgo o probabilidad del individuo, hogar o comunidad de ser herido, lesionado o dañado ante cambios o permanencia de situaciones internas y externas" (pág. 8), en el ámbito educativo, económico, social, físico. A partir de ello se determinará si existe vulnerabilidad social en el contexto de pandemia COVID-19 y si las políticas públicas implementadas por el gobierno estatal y federal con la beca Benito Juárez y la App SIDEMS coadyuvan a la reducir la vulnerabilidad educativa y social para que los estudiantes concluyan satisfactoriamente sus estudios este ciclo escolar 2020- 2021. 


\subsection{Desarrollo}

En México la Educación Media Superior (EMS) y el Sistema Educativo Nacional (SEN) en México, tienen multiplicidad de retos y se ha vuelto más complejo lograr los indicadores de calidad establecidos, ya que actualmente los sistemas educativos en el mundo se encuentran transitando de forma abrupta, de una educación presencial al paradigma de la educación virtual, por la emergencia sanitaria de la pandemia COVID-19, por lo cual se tienen que desarrollar estudios e información del estado que guarda la implementación de la Reforma Integral de la Educación Media Superior (RIEMS) y la operación del Marco Curricular Común (MCC) donde uno de los desafíos es que los docentes organicen y planifiquen didácticamente sus clases virtuales, apegándose a los estándares de calidad establecidos, sin perder de vista el fomento de competencias y guiar a los estudiantes mediante las plataformas y las nuevas tecnologías de la información y la comunicación.

Esto abre una brecha para romper paradigmas de la enseñanza presencial, donde los profesores enfrentan múltiples desafíos relacionados con el dominio efectivo de las diferentes herramientas tecnológicas, que sustentan las nuevas formas de enseñar a aprender, lo cual no solo implica "hablar de equipos, computadoras, dispositivos y programas: es la oportunidad de reflexionar acerca de cómo estamos pensando en la educación y de qué manera los jóvenes y docentes aprenden y enseñan" (UNESCO, 2014, pág. 17), también exige analizar las exigencias del curricular escolar en el ámbito de la educación a distancia, en función de asegurar la cobertura y calidad de la infraestructura tecnológica de toda la comunidad educativa, que permita potenciar el manejo de grupos a distancia, en el ámbito de los procesos de enseñanza y de aprendizaje virtuales, de forma pertinente e innovadora.

Así mismo otro desafío a enfrentar es la equidad digital, en función de garantizar la igualdad de oportunidades de inclusión a los escenarios educativos en esta emergencia sanitaria, a los estudiantes de escasos recursos o que se encuentran en crisis económica, por lo que urge una política pública para atender estas problemáticas relacionadas con la brecha digital (Consejo Nacional de Evaluación, 2020), como estrategias que favorezca la cobertura de este servicio en zonas vulnerables, en particular en situaciones de emergencia, por ello se deben tomar acciones preventivas con base en los programas sociales que el gobierno federal lleva a cabo.

En este ámbito es importante destacar, según los fundamentos emitidos por Albalá e Ignacio (2020) en los contextos de pandemia, las exigencias en el confinamiento de la población: " la brecha digital que se presenta en los hogares más desfavorecidos se ve incrementada, debido a que son estas familias las que cuentan con menores recursos materiales, sociales y culturales para poder hacer frente a estas situaciones" (pág. 179). También evidencian las limitadas condiciones económicas que prevalecen en este porcentaje de la población mexicana, en particular en aquellos que pertenecen a contextos pobres, vulnerables, entre los que se puede precisar; a los estudiantes indígenas, migrantes y de clase media, entre otros. De igual forma está transición abrupta, a un sistema exclusivamente virtual, también han puesto de manifiesto que las instituciones educativas no tienen la capacidad académica y curricular para hacer frente a estos desafíos.

Es importante precisar que The World Economic Forum (The World Economic Forum S. I., Sustainable Development Goal 04: Quality Education, 2020), establece dentro de las tendencias para tener una Educación de Calidad, que se debe considerar una formación post secundaria pertinente, que fomente habilidades para el empleo, la innovación y competencias para la cuarta revolución industrial, considerando las perspectivas de los jóvenes, la igualdad de género y el envejecimiento de la población por ello la EMS, tiene retos para la educación del siglo XXI y por lo cual es necesario analizar la influencia de la RIEMS para determinar los efectos en los aprendizajes de los estudiantes de este nivel educativo en función de mejorar la práctica docente que se da al interior de las aulas. 
Por lo que en la EMS, se debe considerar en el ámbito de la planificación curricular, como opción alternativa viable, el diseñar una modalidad educativa a distancia, como un proceso continuo de adaptación y reinvención que aproveche las máximas capacidades que ofrece el uso efectivo de las TIC en los procesos educativos, todo en correspondencia con las condiciones del contexto socioeducativo, en función de generar estrategias curriculares flexibles que den respuesta a los desafíos de los estudiantes y docentes, ante condiciones emergentes, en función de asumir esta modalidad con una visión prospectiva, como la única opción para hacer frente a las adversidades epidemiológicas y mantener la continuidad de los procesos académicos, en las diferentes modalidades de la oferta. Por lo que se requieren cambios disruptivos de cara hacer frente a la diversidad de problemáticas materiales, sociales y culturales, donde las tecnologías están marcando nuevas formas de gestionar los aprendizajes (García, 2019).

La educación ha sido una herramienta para mejorar el desarrollo humano, tener mayor acceso a un trabajo bien remunerado y mejorar la calidad de vida de países que se encuentran aún en desarrollo y desventaja económica. Organismos internacionales están preocupados por los resultados de pruebas internacionales como PISA (INEE, 2017) donde se muestran resultados por debajo de la media, en los que se evidencia que existe una crisis de aprendizaje en áreas de conocimiento como matemáticas y comprensión lectora que son competencias genéricas, establecidas en la RIEMS.

\subsection{Reforma, vulnerabilidad social y política pública.}

La RIEMS ha avanzado de forma gradual hacia la implementación del MCC y el modelo basado en competencias en las aulas de bachillerato, que coadyuvaron a la mejora de los aprendizajes en la EMS, sin embargo en las condiciones actuales de confinamiento vividas por la pandemia del COVID-19, se ha transitado de clases presenciales a clases virtuales, apoyadas en el uso de herramientas tecnológicas con sesiones síncronas y asíncronas, en las que las actividades escolares se trasladaron a los hogares, sin considerar estrategias adaptativas e incluyentes, en las que se consideran las potencialidades de la modalidad b-learning y la atención personalizadas para atender e incluir a los estudiantes de contextos más desfavorecidos, pues en las condiciones de carencia y precariedad económica, social, de salud pública que se vive en este momento en México y el mundo hace que se cuestione a la educación desde diferentes aristas. Por ello a lo largo de estas reflexiones se consideran los retos actuales que la educación hoy tiene, ya que la vulnerabilidad social surge como una variable más del concepto de la pobreza multidimensional de acuerdo con Pizarro (2001, pág. 12, citado por Rosas Arellano y Bernal, 2019).

En este ámbito el concepto de vulnerabilidad social es analizado desde diferentes connotaciones en base a las aportaciones del Banco Mundial y la ONU, este término tomó relevancia en los años noventa para "prevenir y sobrellevar los efectos que los desastres naturales generaban", posteriormente Foschiatti (2007), señala que "ésta definición se refiere al deterioro de la calidad de vida, la inestabilidad económica, pobreza, la fragmentación social, la precariedad a partir de los sistemas defensivos" (pág. 15), lo anterior nos deja ver que son situaciones que se tornan en riesgo para el desarrollo humano de los individuos. Asimismo, conforme ha ido evolucionando el estudio de la vulnerabilidad se han desarrollado enfoques, clasificaciones las cuales ayudan a ver que ciertos grupos dentro de la sociedad tienden a tener características de vulnerabilidad y que parte del estudio de la pobreza multidimensional en México (Lara Espinosa, 2015).

Existen diversos estudios de vulnerabilidad social que abordan el tema desde un punto de vista distinto a lo que se pretende explicar en este trabajo, es diferente por el contexto, el grupo objetivo y el ambiente, así lo señalan Sánchez-González y Egea-Jiménez (2011) en su estudio de vulnerabilidad social para investigar desventajas socioambientales en los adultos mayores, donde identificaron que la geografía en la que se encuentran inmersas estas poblaciones y las características territoriales juegan un papel importante para identificar a los más desprotegidos. 
Así mismo Macías M. (2016), estudia la vulnerabilidad social a partir de la dimensión de los fenómenos climáticos en donde analiza la indefensión de la población por los tornados en de la ciudad de México, y como la falta de preparación del personal del área de meteorología para identificar este tipo de sucesos ambientales pueden impactar en la predicción oportuna para apoyar a las personas más vulnerables a partir del suceso del 1 de junio del 2012.

Otra investigación sobre vulnerabilidad social con enfoque educativo es mostrada por Díaz López y Pinto Loria (2017), donde se explica como una situación multifactorial que afecta a los estudiantes a lo largo de su trayectoria académica y que puede desembocar en el fracaso escolar. En esta investigación se analizaron trece casos de estudiantes del nivel secundaria en Yucatán-México con base en un inventario de riesgo suicida para adolescentes, de los resultados arrojados se identificaron que existen casos de cutting, alcoholismo, problemas de desintegración familiar. Finalmente se comenta que a pesar de que la escuela ha hecho esfuerzos por apoyar, no existe un plan ni atención para este tipo de alumnos.

En otros trabajos se ha descrito el impacto negativo de la pobreza y vulnerabilidad en los estudiantes y las familias utilizando metodologías cuantitativas y descriptivas (Durán \& González, 2013), en donde se muestra que la economía, desempleo e ingresos por debajo del salario mínimo condicionan a los alumnos y los ponen en desventaja para concluir su escuela. Sin embargo, ahora en este estudio existe un factor adicional que es la pandemia mundial COVID-19.

Murillo y Duk (2020) analizan las brechas que se están generando a partir de la pandemia COVID-19 en los hogares de los estudiantes y cómo el confinamiento puede tener efectos negativos en lo económico y social, así como la falta de acceso a internet, el poco manejo de plataformas virtuales por parte de los estudiantes y docentes.

Otras acepciones que dan sustento a este trabajo es la implementación de las políticas públicas, ya que éstas son retomadas para atender o focalizar los grupos con mayor vulnerabilidad social, pobreza y desigualdad, en este caso el grupo objetivo de la beca Benito Juárez y usuarios de la App SIDEMS son los estudiantes de la educación media superior en riesgo de exclusión social, todo ello con el propósito de reducir el rezago educativo y prevenir el abandono escolar en esta pandemia.

En este sentido es necesario abordar ¿qué son las políticas públicas?, del cual emergen diferentes visiones, siendo un término polémico al interior de las ciencias políticas ya que al inicio surgieron diversas formas de abordarse, de definirse y de analizarse. Subirats, Knoepfel, Larrue, \& Varone (2008) refieren que según las aportaciones de Mény \& Thoenig (1989) es importante analizar las políticas públicas y tiene que ver con "el estudio de acción de los poderes públicos en el seno de la sociedad" (pág. 10), desde donde emergen la capacidad directiva de los gobiernos en respuesta a los problemas sociales, en función mitigar los problemas de la pobreza, mejora de la educación y sana alimentación. Sin embargo, su utilización en su inicio era analizado desde diferentes perspectivas (Wright, Lerner, \& Lasswell, 1952).

Así mismo Aguilar Villanueva (1992) en su libro sobre la hechura de la política, analiza a diversos autores como Subirats, Knoepfel, Larrue, \& Varone (2008) y Ham (1986) quienes señalan que el término política aborda diversos significados desde una actividad gubernamental, una situación social, propuesta de acción específica, normas o como resultado de una actividad gubernamental. Finalmente determina que la política pública es un curso intencional acción, sigue un actor o un conjunto de actores al tratar un problema o asunto de interés, en función de resolver diversas situaciones problemáticas. Por otra parte, es importante mencionar que siempre está presente el clientelismo electoral en los programas sociales como estrategias para ganar el poder; ésta es otra mirada que siempre está presente en el diseño de políticas públicas y que más allá de resolver problemas públicos atiende intereses de grupos en pugna. 
Por su parte Lindblom (1994) describe el estudio y análisis de la política pública, refiere que el mismo tiene cuatro reglas las cuales parten de una visión que promete desarrollar o diseñar políticas públicas; en primer lugar, deben ser apegadas a la realidad, al contexto y deben responder a un análisis costo beneficio dejando de lado los ideales del analista; segundo se debe ver la viabilidad en cuanto al costo y si son incompatibles para la población; en tercer lugar el analista de políticas públicas debe formarse no sólo para él, sino para enfocarse en escribir para quién toma las decisiones en el gobierno y adecuarse a los problemas; finalmente el cuarto lugar tiene un enfoque en base al asunto público, que implica la reformulación de un problema junto con una serie de alternativas posibles, que culminan en una recomendación al encargado de tomar decisiones.

Reflexionar sobre estos términos, precisa que desde una visión analítica, reflexiva y prospectiva se generen políticas públicas alineadas a dar respuesta a situaciones de emergencia, que exijan de un confinamiento global, en particular en el ámbito educativo, como referentes para generar estrategias viables de inclusión educativa en el ámbito de la flexibilidad académica e institucional. Por lo que se requiere analizar estrategias y modelos teóricos que sustenten las posibilidades de organizar el currículo para dar respuestas óptimas a los desafíos presentes y futuros en situaciones de confinamiento.

\section{Metodología}

Este estudio tiene un enfoque de corte cuantitativo exploratorio, no experimental, donde se aplicaron técnicas de estadística descriptiva como primer acercamiento y en una segunda fase se implementarán de corte inferencial. Se elaboró un cuestionario con base en los tipos de vulnerabilidad social de Wilches-Chaux (1993), citado por Rosas Arellano \& Bernal (2019), utilizando la plataforma de formularios de las aplicaciones de Google, y se envió al correo electrónico de los estudiantes. Se contó con el apoyo de dos académicos de los planteles escogidos. La muestra de la población fue aleatoria, en la que participaron 125 sujetos de estudio de diferentes semestres en el ciclo escolar 2020-2021.

La encuesta, sobre vulnerabilidad social, abarco los siguientes aspectos: carencias sociales en la pandemia, recursos para el aprendizaje en casa, situación de amenazas, conclusión del ciclo escolar y apoyo económico.

A continuación, se reportan y analizan los resultados de la encuesta, aplicada a 125 estudiantes de la educación media superior en Hidalgo en los planteles COBAEH San Agustín Tlaxiaca y COBAEH Tepehuacán de Guerrero, todo fue respondido a través del cuestionario aplicado con el apoyo de Google Forms; los resultados se exportaron a Google Sheets, para realizar la selección, traducción y codificación de los datos, en función de generar las estadísticas descriptivas y las gráficas de los resultados

\section{Resultados}

La figura 1. recoge información porcentual de los 125 estudiantes de los cuales el $53.6 \%$ son mujeres y el $46.4 \%$ son hombres, la distribución de edades se encuentra en un rango de 15 a 20 años. 
Figura 1

Distribución porcentual de Sexo

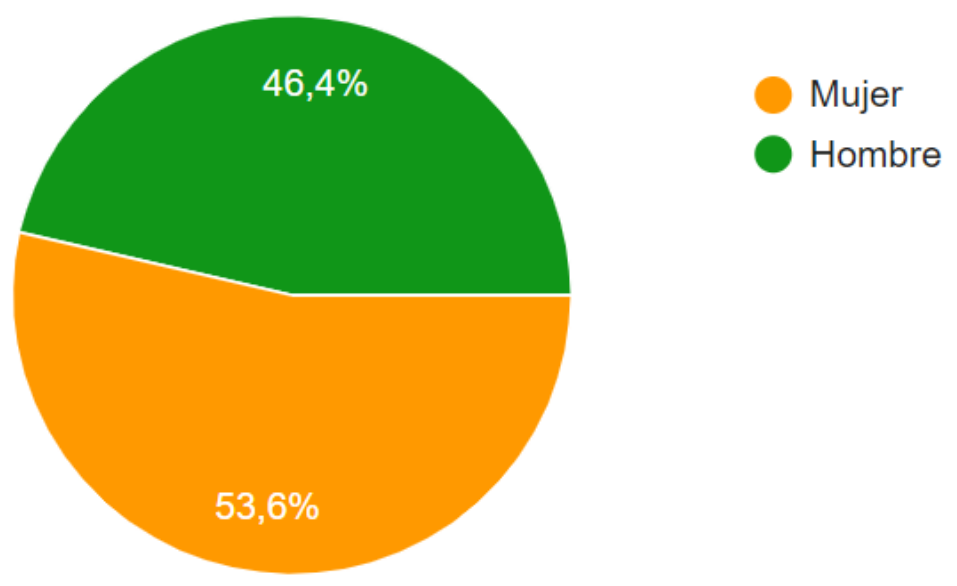

Fuente: Elaboración propia

Con relación a la pregunta sobre las carencias sociales, a partir de la emergencia sanitaria en la familia, se presentan en la figura 2 los porcentajes de vulnerabilidad social que ha revelado el estudio.

Figura 2

Carencias sociales en la pandemia

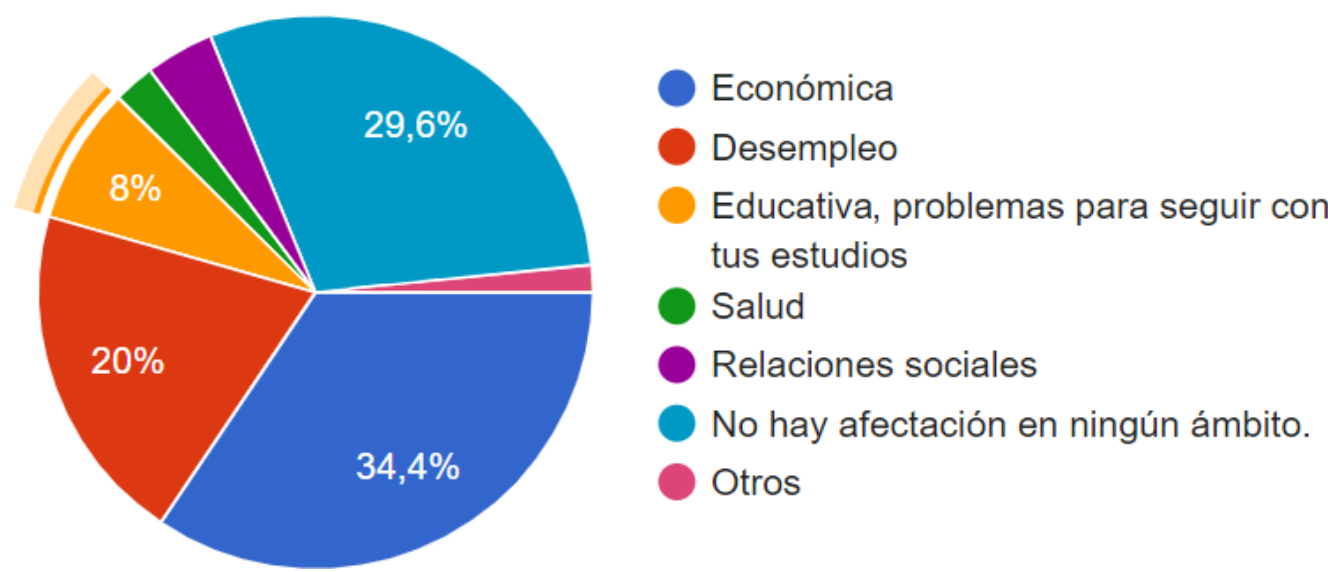

Fuente: Elaboración propia

Los resultados expresados en la figura 2 muestran que a partir de la pandemia COVID-19 las familias de los estudiantes de los planteles COBAEH, San Agustín Tlaxiaca y Tepehuacán de Guerrero, presentaron las siguientes carencias sociales: $34.4 \%$ económica, el $20.0 \%$ desempleo, $8.0 \%$ tienen problemas para continuar con sus estudios, en menor proporción han presentado alguna dificultad de salud y con las formas de relacionarse socialmente, por otra parte el $\mathbf{2 9 . 6 \%}$ no han tenido alguna problema o carencia. Por lo anterior se infiere que la familia de los estudiantes tiene vulnerabilidad económica, de ingresos y falta de acceso al trabajo.

Referente a la política aprende en casa se identificaron los siguientes problemas relacionados con la App SIDEMS, acceso a internet, acceso a una computadora o teléfono móvil. En la figura 3, se presentan los principales hallazgos. 
Figura 3

Recursos para el aprendizaje en casa

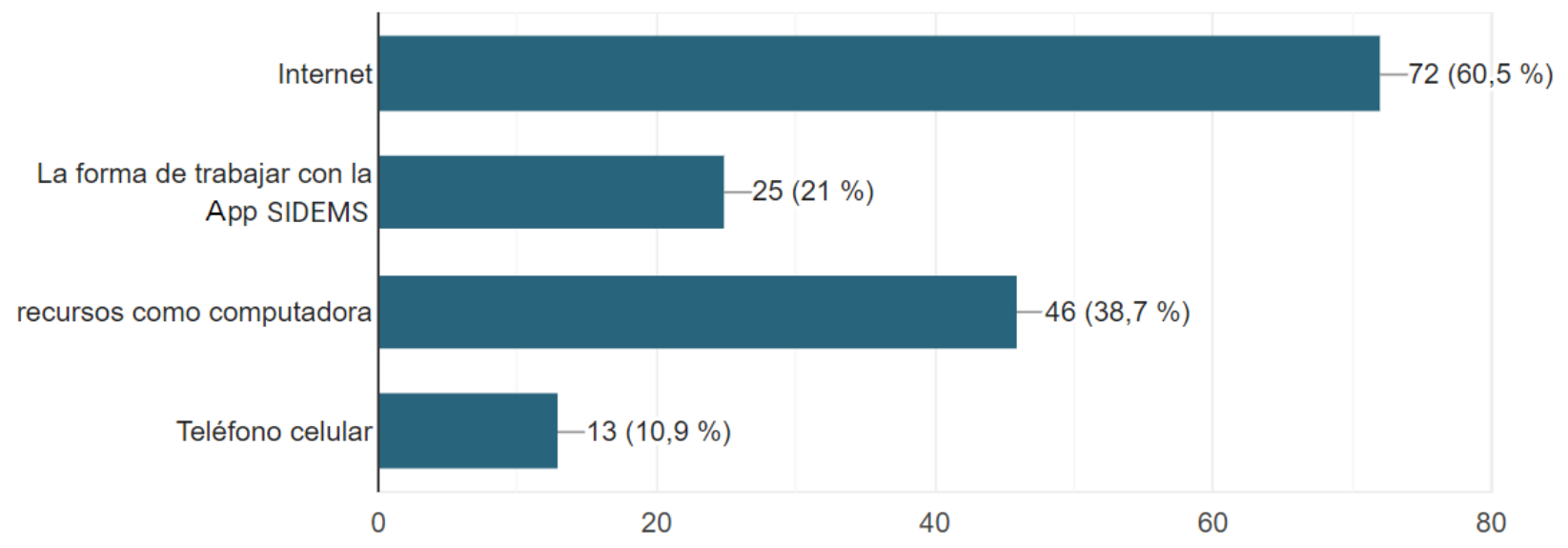

Fuente: Elaboración propia

En este sentido los estudiantes, indicaron que el $60.5 \%$ ha tenido alguna dificultad de acceso con el internet, 21.1\% ha tenido problema para trabajar con la App de SIDEMS, el 38.7\% no cuenta con recursos como computadora y el $10.9 \%$ con teléfono celular.

Así mismo respecto a la pregunta tres, donde se indaga sobre alguna situación que ponga en riesgo la convivencia familiar, integridad física, economía o salud, se presentan los principales resultados en la figura 4.

Figura 4.

Porcentaje de vulnerabilidad social

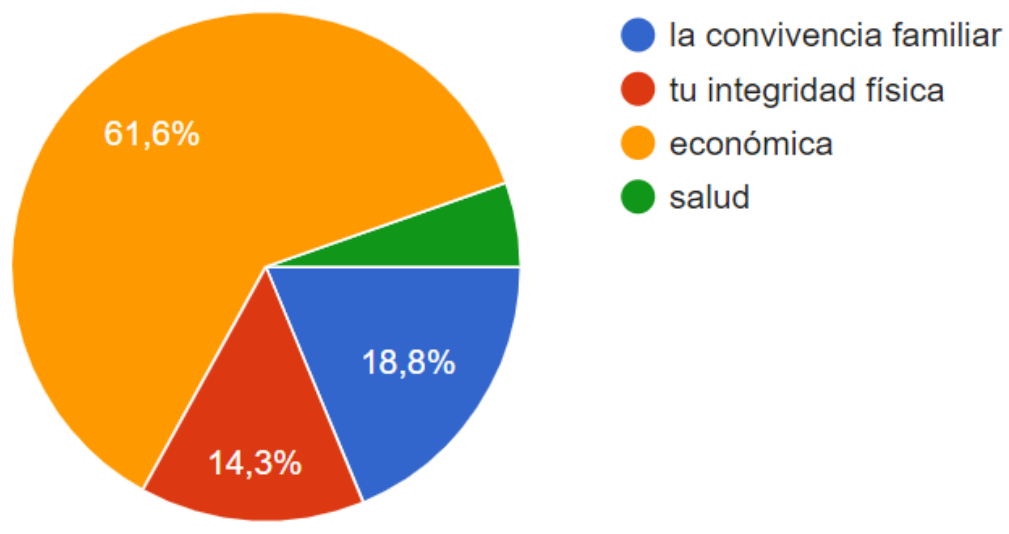

Fuente: Elaboración propia

Por lo que se puede identificar a partir de las aportaciones de los estudiantes durante su estancia en casa, que la problemática de mayor riesgo que los ha afectado es la economía (61.6\%), seguida de la convivencia familiar (18.8\%), el 14.3\% la integridad física, y en menor proporción las relacionadas con la salud (4.5\%). y, lo cual condiciona de un análisis contextualizado en cada plantel, en función de generar acciones viables que apoyen a los estudiantes, ante las adversidades que están enfrentando.

En este mismo orden de análisis se considera en la figura 5 la pregunta relacionada sobre si existe algún riesgo de perder el ciclo escolar en curso, en la que se obtuvieron los siguientes hallazgos. 
Figura 5

Porcentaje de riesgo para concluir satisfactoriamente

el ciclo escolar 2020- 2021

Con la Pandemia COVID 19, y el desarrollo de las clases en linea /en tv/ plataforma existe riesgo que :

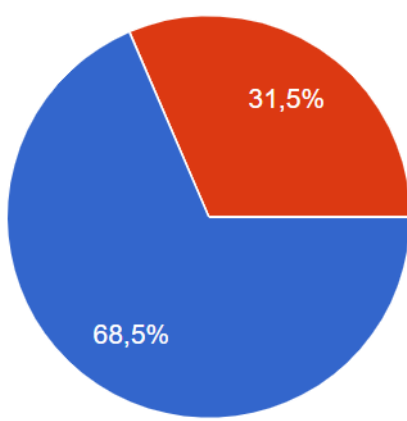

no concluyas satisfactoriamente tu periodo escolar

si concluyas satisfactoriamente tu periodo escolar

Fuente: Elaboración propia

De acuerdo con los resultados anteriores, el $68.5 \%$ de los estudiantes consideran tener riesgo de no concluir satisfactoriamente su ciclo escolar en tiempo y forma, por otra parte, el $31.5 \%$ refieren que tienen las posibilidades de concluir de manera satisfactoria sus estudios.

Fue incluida una pregunta para conocer cuántos de los estudiantes de la muestra han sido beneficiaros con la beca Benito Juárez, la cual esta focalizada para atender a alumnos en situación de pobreza. La figura 6 nos muestra que la gran mayoría no tiene acceso a este apoyo con un $86.8 \%$, mientras que el $13.2 \%$ de los estudiantes si cuenta con esta beca.

Figura 6

Porcentaje de alumnos que cuentan con la beca Benito Juárez

\section{¿Cuentas con el apoyo de la beca Benito Juárez?}

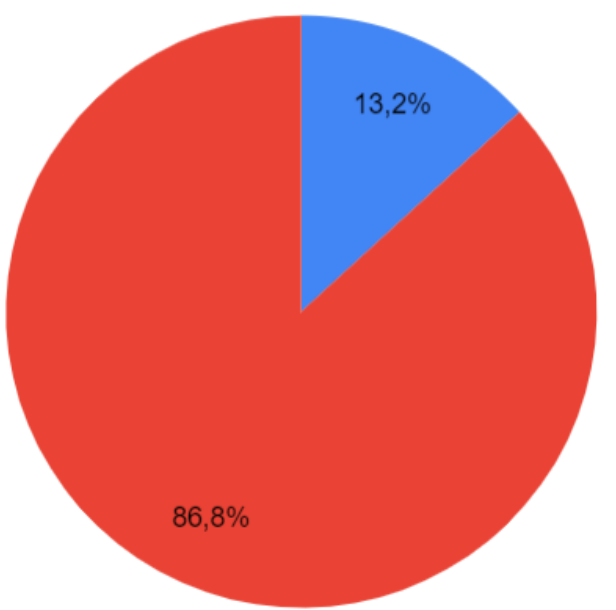

Fuente: Elaboración propia

En este mismo orden de análisis en la pregunta sobre el apoyo de beca y el impacto de ésta en la economía familiar, en la figura 7, se refieren los siguientes resultados. 


\section{Figura 7}

Distribución porcentual de apoyo beca y economía familiar.

\section{Ha ayudado a tu economía familiar:}

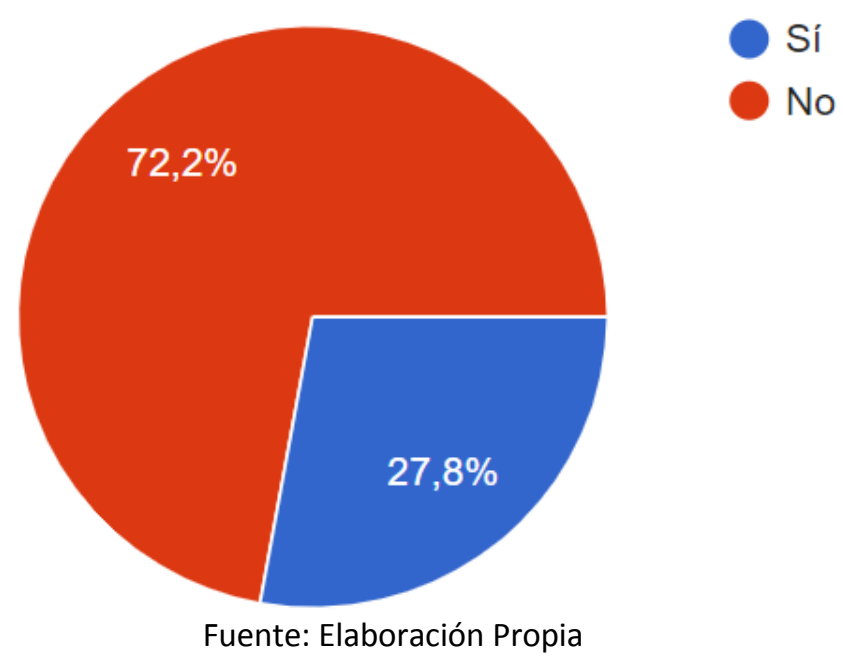

A partir de los resultados anteriores, se precisa que de los 125 estudiantes que conforman la muestra de estudio, sólo 79 alumnos tienen beca lo que equivale al $63.2 \%$, de los cuáles el $72.2 \%$ consideran que este apoyo no ha ayudado a su economía familiar y el $29.5 \%$ si cree que la beca ha coadyuvado a la economía familiar.

Sin embargo, la muestra fue aleatoria y no se incluye a la población total, por lo que la estimación es acertada, pero hay que hacer una comparación a través de las estadísticas generadas por parte de las autoridades educativas, con propósito de analizar si existe datos en torno a estos indicadores, que reflejen las condiciones económicas de las familias, para poder establecer correlaciones al respecto. Lo anterior indica que las políticas sociales de apoyo económico y focalización a este grupo vulnerable, si se está llevando en el ejercicio del presupuesto para reducir el índice de abandono escolar por esta situación.

En un segundo momento de este análisis es importante señalar que en el cuestionario fue considerada una pregunta abierta, con el propósito de conocer las percepciones de los estudiantes sobre la vulnerabilidad social y educativa a partir de esta emergencia sanitaria y al respecto, las principales aportaciones referidas por los estudiantes son las siguientes:

-Aún existen personas que no cuentan con aparatos electrónicos e internet (Estudiante 1).

-La educación en casa si deja algún aprendizaje por ejemplo nos enseña a ser responsables, y ser más autónomos e independientes para aprender por nuestra propia cuenta, pero también nos afecta ya que algunos no tenemos internet para poder trabajar (Estudiante 2).

- Es difícil comprender así los trabajos, pues no tenemos acceso a internet para poder buscar información complementaria (Estudiante 3);

-Hay algunas materias que no les entiendo y se me dificultan más... química se me complica mucho, pero vamos interactuando entre los miembros del equipo y con el docente (Estudiante 4); 
-Las clases son buenas, pero no todos tienen las posibilidades de poder enviar los trabajos a tiempo y los docentes no son flexibles con ello, pues nos dicen que tienen enviar evidencias a sus jefes (Estudiante 5).

-Aprendo a manejar otro tipo de aplicaciones y la verdad que me he ido probando, a veces no sabemos hasta donde podemos llegar, tengo la posibilidad de tener una buena conexión a internet y eso ha sido lo que me ha favorecido poder cumplir con las actividades solicitadas (Estudiante 6).

A partir de estos criterios emitidos por los estudiantes se evidencia la incapacidad institucional de poder atender de forma inclusiva, las demandas y necesidades de los estudiantes en situaciones de la emergencia sanitaria, provocada por el COVID-19, pues la mayoría refiere no contar con el acceso a internet, lo evidencía las situaciones de vulnerabilidad en las que están inmersos, lo cual se puede validar a través de las estadísticas emitidas por el Instituto Nacional de Estadística y Geografía, INEGI-IFT (2020), cuando refiere que en México, solo el 56.4\% de los hogares tienen acceso a internet, lo cual trasciende a desenmascarar las diferencias existentes en el país.

En tal sentido es importante señalar que los actores involucrados en la aplicación de la política pública, federal (Becas Benito Juárez) y estatal (App SIDEMS), en los planteles objetos de estudio, tienen como reto analizar de forma contextualizada las condiciones socioeconómicas de la comunidad educativa para atender la mayor cantidad posible de estudiantes que se encuentren en situaciones de riesgo y sean promotores y acompañantes de las transformaciones educativas, necesarias para estás modalidades que se han llamado a distancia, hibridas, b-learning, virtuales, etc; las cuales requieren que los estudiantes cuenten con recursos tecnológicos que apoyen sus aprendizajes.

Por lo que las situaciones de desigualdad social que prevalecen en la población objeto de estudio, donde el acceso a internet tiene una cobertura limitada, se requiere de que las políticas públicas, orientadas a apoyar a los estudiantes del bachillerato sean revisadas, en función de ampliar su impacto en un mayor número de beneficiarios, pues en gran medida están influyendo negativamente en la construcción de entornos de aprendizajes adaptativos e incluyentes, que impacten a toda la población estudiantil.

\section{Conclusiones}

Este estudio es un acercamiento para conocer su impacto de las políticas públicas, relacionadas con la asignación de recursos a los estudiantes y en particular a partir de la pandemia COVID-19, por lo que es necesario que se evalúen sus efectos y se dé un seguimiento para realizar mejoras en la operación de ambas. En base a lo anterior se concluye que la vulnerabilidad social de los estudiantes de los planteles de Tepehuacán de Guerrero y San Agustín Tlaxiaca, está afectada por la condición socioeconómica de sus hogares y la falta de empleo en las familias, lo cual pone en riesgo su permanencia en este nivel educativo, ya que si entendemos que la vulnerabilidad parte del estudio de la pobreza multidimensional esto vuelve más complejo el panorama de la conclusión en el ciclo escolar, lo cual trasciende a la exclusión educativa.

A través de esta exploración diagnóstica, se asevera que la política pública implementada para apoyar a los estudiantes con becas de este nivel educativo no es una estrategia emergente por la pandemia, ya se encontraba en operación por el gobierno federal. Sin embargo, la App de SIDEMS es una estrategia emergente por el gobierno del estado de Hidalgo, ambas han pretendido atenuar la brecha digital en estudiantes en situaciones económicas vulnerables, para poder acceder a las nuevas formas de aprendizaje mediadas por el uso de la red, sin que aún por el momento, se haya logrado un impacto relevante para este grupo vulnerable, en gran medida debido a la velocidad con la que se han venido exigiendo cambios en esta nueva normalidad a causa de la pandemia.

De igual forma es importante señalar que se encuentra en riesgo de aumentar el índice de desarrollo humano para los próximos años en el estado de Hidalgo, por lo que es necesario revisar el acceso a internet y mejorar la 
aceptación y uso de la App SIDEMS ya que algunos estudiantes están inmersos en estas problemáticas, lo cual puede condicionar situaciones de pobreza extrema que los excluya de las posibilidades de continuar con sus estudios en este nivel educativo.

Otro rubro importante que se debe analizar en el ámbito de las instituciones educativas es la focalización de más recursos para ampliar la población objetivo de estudiantes beneficiados con becas y así brindar la oportunidad de terminar satisfactoriamente su trayecto formativo en el bachillerato. Por ello se requiere dar seguimiento a las políticas de aprendizaje en casa, el desempeño y capacitación a docentes para mejorar sus clases en línea, así como ver el estado que guardan los resultados de los aprendizajes de los estudiantes a partir de las exigencias y demandas de la RIEMS en función de reducir el rezago educativo y conducir la toma de decisiones que impacten en la generación y/o reformulación de las políticas educativas orientadas a apoyar a los estudiantes, como garantía de apoyar estratégicamente su trayectoria formativa, particular en la educación media-superior.

\section{Agradecimientos}

La Maestra Adriana Arlette Ibarra Ramírez le agradece al CONACYT el apoyo otorgado a través de la Beca para Estudios de Doctorado, con número de solicitud 2019-000037-02NACF-25620.

Este trabajo forma parte de los resultados preliminares de la investigación que se desarrolla en el Doctorado en Políticas Públicas de la Universidad Autónoma del Estado de Hidalgo, programa que pertenece al Padrón de Posgrado de CONACYT.

\section{Referencias bibliográficas}

Albalá, M., \& Ignacio, J. (2020). La brecha socioeducativa derivada del Covid-19: posibles abordajes desde el marco de la justicia social (Número Especial ed., Vol. L). México: RLEE Nueva Época. https://rlee.ibero.mx/index.php/rlee/article/view/101/475

Aguilar Villanueva, L. F. (1992). La Hechura de las Políticas. Estudio Introductorio. México. Editorial Miguel Ángel Porrúa Grupo Editorial.

Becasmx (2020). Becas del nivel medio superior Benito Juárez. https://becasmx.com/becas-benito-juarez/

Consejo Nacional de Evaluación de la Política de Desarrollo Social (CONEVAL). (2020). Evaluación de la Política Social en el contexto del COVID-19. https://www.coneval.org.mx/Evaluacion/IEPSM/Paginas/Politica_Social_COVID-19.aspx

Busso, G. (2001). Vulnerabilidad social: nociones e implicancias de políticas para Latinoamérica a inicios del siglo XXI. Santiago de Chile: CEPAL.

Consejo Nacional de Evaluación, M. (2020). Evaluación de la Política Social en el contexto del COVID-19. Evaluación de la Política Social en el contexto del COVID-19. Obtenido de https://www.coneval.org.mx/Evaluacion/IEPSM/Paginas/Politica_Social_COVID-19.aspx

Coordinación Nacional de Becas para el Bienestar, B. J. (2020). Becas Benito Juárez. Becas Benito Juárez. Gobierno de México. Obtenido de https://www.gob.mx/becasbenitojuarez

Diario Oficial de la Federación, SEP. (2008). Sistema Nacional de Bachillerato en un marco de diversidad ACUERDO número 442, por el que se establece el sistema nacional de bachillerato en un marco de diversidad. http://dof.gob.mx/nota_detalle.php?codigo=5061936\&fecha=26/09/2008 
Díaz López, C., \& Pinto Loria, M. (2017). Vulnerabilidad educativa: un estudio desde el paradigma socio crítico / Educational Vulnerability: A study from the socio-critical paradigm. Praxis Educativa, 21, 46-54. doi:10.19137/praxiseducativa-2017-210105

Durán, L. I., \& González, R. C. (2013). Pobreza y vulnerabilidad. Factores de riesgo en el proceso educativo. Contextos Educativos. Revista de Educación, 55-72.

Dye, T. R. (2002). Understanding Public Policy. Prentice Hall College Div. https://books.google.com.mx/books?id=R5ApAQAAMAAJ

Espinosa, D. L. (2015). Grupos en situación de vulnerabilidad. Comisión Nacional de los Derechos Humanos. México. Editorial CNDH. https://books.google.com.mx/books?id=L1\\{p\}\{o\}\{Q\}EACAAJ

Franco Corzo, J. (2013). Diseño de políticas públicas (3a ed.). México: IEXE Editorial.

Foschiatti, A. M. (2007). Aportes conceptuales y empíricos de la vulnerabilidad global. RIUNNE - Repositorio Institucional de la Universidad Nacional del Nordeste.

García, L. (2019). Necesidad de una educación digital en un mundo digital. Revista Iberoamericana de Educación a Distancia, 9-22. doi:http://dx.doi.org/10.5944/ried.22.2.23911

Gúzman Gómez, C. (2020). Daños y saldos de la pandemia por covid-19 en escuelas vulnerables: el caso de los Telebachilleratos Comunitarios en México. México. Editorial. Centro Regional de Investigaciones Multidisciplinarias-UNAM. http://www.comie.org.mx/v5/sitio/2020/08/04/danos-y-saldos-de-lapandemia-por-covid-19-en-escuelas-vulnerables-el-caso-de-los-telebachilleratos-comunitarios-en-mexico/

Ham, C. (1986). B. Hogwood and L. Gunn, Policy Analysis for the Real World, Oxford University Press, Oxford, 1984. x 289 pp. $£ 16.00$, paper £8.95. Journal of Social Policy, 15, 132-133. doi:10.1017/S0047279400023205

INEE. (2017). México en PISA 2015. México en PISA 2015. Unidad de Evaluación del Sistema Educativo Nacional, INEE. Obtenido de https://historico.mejoredu.gob.mx/publicaciones/mexico-en-pisa-2015/

INEGI-IFT. (2020, 2). Encuesta Nacional sobre Disponibilidad y Uso de Tecnologías de la Información en los Hogares,(ENDUTIH) 2019. Comunicado de Prensa - INEGI, 103/20, 11-13. Retrieved from https://www.inegi.org.mx/contenidos/saladeprensa/boletines/2020/OtrTemEcon/ENDUTIH_2019.pdf

Jenkins, W. I. (1978). Policy Analysis: A Political and Organisational Perspective. Editorial M. Robertson. https://books.google.com.mx/books?id=7k15mgEACAAJ

Kraft, M. E., y Furlong, S. R. (2019). Public Policy: Politics, Analysis, and Alternatives. Washington, D.C: CQ Press

Lara Espinosa, D. (2015). Grupos en situación de vulnerabilidad. Comisión Nacional de los Derechos Humanos. Obtenido de https://books.google.com.mx/books?id=L1 \\{p\}\{o\}\{Q\}EACAAJ

Lindblom, C. E. (1994). La investigación social para la elaboración de políticas: quién la necesita y para qué. Gestión y Política Pública. México. Editorial Miguel Ángel Porrúa Grupo Editorial.

Macías M., J. M. $(2016,6)$. Vulnerabilidad social en la Ciudad de México frente a tornados. Revista mexicana de sociología, 78, 257-284. Retrieved from http://www.scielo.org.mx/scielo.php?script=sci_arttext\&pid=S0188-25032016000200257\&nrm=iso

Mény, Y., \& Thoenig, J.-C. (1989). Politiques publiques. Presses universitaires de France (Paris). Obtenido de http://catalogue.bnf.fr/ark:/12148/cb36640124b 
Murillo, F. J., \& Duk, C. $(2020,6)$. El Covid-19 y las Brechas Educativas. Revista latinoamericana de educación inclusiva, 14, 11-13. Retrieved from https://scielo.conicyt.cl/scielo.php?script=sci_arttext\&pid=S0718$73782020000100011 \&$ nrm=iso

Organización para la Cooperación y el Desarrollo Económicos (OCDE). (2020). A helping hand: Education responding to the coronavirus pandemic. Publicaciones de la OCDE, págs. 1-2. https://oecdedutoday.com/education-responding-coronavirus-pandemic/

Organización de las Naciones Unidas, (ONU). (2015). Agenda 2030 para el Desarrollo Sostenible. Agenda 2030 para el Desarrollo Sostenible. http://dof.gob.mx/nota_detalle.php?codigo=5061936\&fecha=26/09/2008

Rosas Arellano, J., y Bernal, A. (2019). El alcance de los enfoques de vulnerabilidad y pobreza para la definición de la población objetivo en programas sociales. Gestión y Política Pública, 28, 351. doi:10.29265/gypp.v28i2.623

Sánchez-González, D., \& Egea-Jiménez, C. (2011, 9). Enfoque de vulnerabilidad social para investigar las desventajas socioambientales: Su aplicación en el estudio de los adultos mayores. Papeles de poblacĩ̃ \textthreesuperiorn, 17, 151-185. Retrieved from http://www.scielo.org.mx/scielo.php?script=sci_arttext\&pid=S1405-74252011000300006\&nrm=iso

Sanz, I., Sáinz, J., y Capilla, A. (2020). Efectos de la Crisis del Coronavirus en la Educación Superior. Madrid: Organización de Estados Iberoamericanos para la Educación. https://oei.org.br/arquivos/informe-covid19d.pdf

SIDEMS (2020). Aplicación SIDEMS https://www.sidems.com/ y https://play.google.com/store/apps/details?id=com.MultiExpo.sidems\&hl=es

Shepsle, K. A., y Serra, G. (2016). Analizar la política. Comportamiento, instituciones y racionalidad. México. Centro de Investigación y Docencia Económicas A.C.(CIDE).

Subirats, J., Knoepfel, P., Larrue, C., y Varone, F. (2008). Análisis y gestión de políticas públicas. Barcelona. Ariel.

The World Economic Forum, W. E. (2020). GLOBAL ISSUE COVID-19 Curation. https://intelligence.weforum.org/topics/a1G0X00000606EHUA0?tab=data

The World Economic Forum, S. I. (2020). Sustainable Development Goal 04: Quality Education. Sustainable Development Goal 04: Quality Education. Strategic Intelligence, World Economic Forum. Obtenido de https://intelligence.weforum.org/topics/a1G0X0000057NO0UAE?tab=publications

Torres Melo, J., y Santander A., J. (2013). Introducción a las políticas públicas. Conceptos y herramientas desde la relación entre Estado y ciudadanía. Bogotá. Editorial IEMP.

UNESCO. (2014). Enfoques estratégicos sobre las TICS en Educación en América Latina y. Santiago de Chile, Chile: OREALC/UNESCO . https://unesdoc.unesco.org/ark:/48223/pf0000223251_spa

Vergara, R. (1993). Decisiones, organizaciones y nuevo institucionalismo. Perfiles latinoamericanos. Vol. 2. №. 3.

Wilches-Chaux, G. (1993). La vulnerabilidad global. Los desastres no son naturales, 11-44.

Wright, Q., Lerner, D., \& Lasswell, H. (3 de 1952). The Policy Sciences; Recent Developments in Scope and Method. The American Political Science Review, 46, 234. doi:10.2307/1950773.

Esta obra está bajo una Licencia Creative Commons Attribución-NoCommercial 4.0 International 